\title{
STEM SI Warp: A Tool for Correcting the Linear and Nonlinear Distortions for Atomically Resolved STEM Spectrum and Diffraction Imaging
}

Yi Wang, Y. Eren Suyolcu, Ute Salzberger, Kersten Hahn, Vesna Srot, Wilfried Sigle and Peter A. van Aken

Stuttgart Center for Electron Microscopy, Max Planck Institute for Solid State Research, Stuttgart, Germany

Specimen and stage drift as well as scan distortions can lead to a mismatch between the true and desired electron probe positions in scanning transmission electron microscopy (STEM) which may result in both linear and nonlinear distortions in the subsequent experimental images. Generally, STEM spectrum imaging (SI) and diffraction imaging (DI) both require much higher electron dose and longer dwell time (pixel exposure time) than STEM imaging. As a consequence, these distortions become more serious in spectrum/diffraction imaging data. In case of lower-magnification, these instabilities may be negligible, but at the atomic resolution level, instabilities combined with long dwell times may create substantial image distortions, i.e. expansion, compression, and/or shearing of the lattice (Figure 1), that limit the interpretability of the results. Currently, popular atomic resolution STEM-SIs acquisition techniques use on-the-fly drift-correction [1] or directly sum up multiple fast-dwell-time SI that have been driftcorrected between separate SIs [2]. More recently, rigid and non-rigid registration techniques aligning multi-frame SIs have also been reported [3-5]. However, these experiments are not yet routine and require advanced registration algorithms to perform the alignment. Moreover, these techniques may not be practical to STEM-DI due to big data handling issues.

In this contribution, we report the development of a software tool [6], written in the Digital Micrograph (DM) scripting language, for post collection correction (off-the-fly) of image distortions in atomically resolved spectrum and diffractive imaging. It can be used to correct two typical image distortions, i.e., linear and nonlinear distortions, as shown in Fig. 1. In the algorithm, the transformation of the coordinate system was performed by using the "warp" function, which uses bilinear interpolation. The warp and image-shift functions work for multi-dimensional data (2D to 4D). With prior knowledge of the crystal structure, the diagnosis of the image distortions was performed on simultaneously acquired ADF images. Subsequently, the calculated image distortion correction can be applied to both the ADF image and the multidimensional dataset, which can be 2D images, i.e., computed elemental maps, reconstructed images, or 3D spectrum imaging data as well as 4D diffraction imaging data.

With three practical examples, i.e., 2-dimensional Ba-doped $\mathrm{La}_{2} \mathrm{CuO}_{4}$, a $\mathrm{NdVO}_{3}$ thin film grown on $\mathrm{NdGaO}_{3}$ (NGO) substrate, and $\mathrm{SrTiO}_{3}$ bulk material, we demonstrate the correction of these linear and nonlinear distortions (Figure 1), feasibility of the script for 4D STEM diffraction imaging (Figure 2), and the application for maximizing the elemental mapping area. This software tool significantly improves the interpretability of distorted STEM spectrum/diffraction imaging data. The software (plugin for DM) is available by request to the authors. For advanced users, the multidimensional warp and offset functions are installed as libraries and can be called externally.

References:

[1] K Kimoto et al., Nature 450 (2007), p. 702.

[2] P Lu et al., Sci. Rep. 4 (2014), p. 3945. 
[3] A B Yankovich et al., Nanotechnology 27 (2016), p. 364001.

[4] J S Jeong, K A Mkhoyan, Microsc. Microanal. 22 (2016), p. 1.

[5] Y Wang et al, Ultramicroscopy 184 (2018), p. 98.

[6] Y Wang et al, Microscopy (2018) https://doi.org/10.1093/jmicro/dfy002
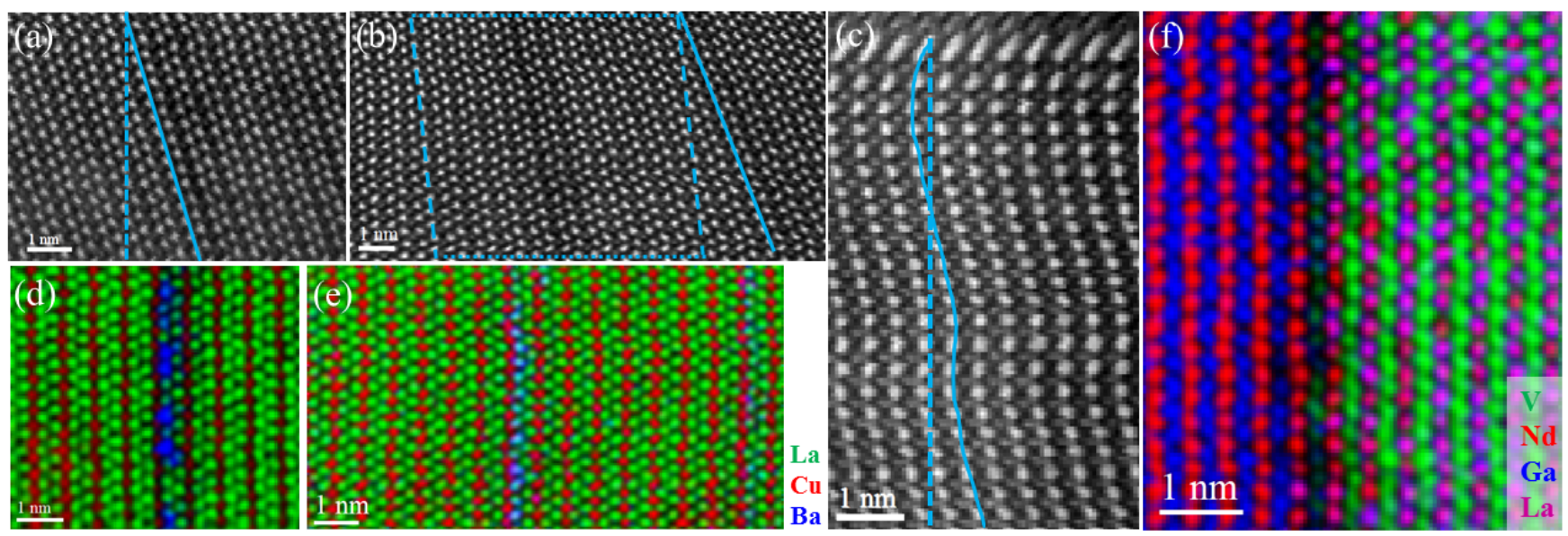

Figure 1. Typical image distortions in spectrum imaging: ( $a, b)$ linear and (c) nonlinear distortions. As can be seen the straight lattice (indicated by the dotted lines) has been distorted into an inclined or curved line. Examples demonstrating the correction of linear and nonlinear distortions: (d) and (e) final corrected and cropped RGB color figure of the overlaid elemental maps, Cu- $\mathrm{L}_{2,3}(\mathrm{Red}), \mathrm{La}-\mathrm{M}_{4,5}$ (Green), and $\mathrm{Ba}_{4,5}$ (Blue), (f) final corrected and cropped RGB color figure of the overlaid elemental maps, Nd-M4,5 (red), V-L 2,3 (green), Ga-L2,3 (blue), and La-M4,5 (magenta).

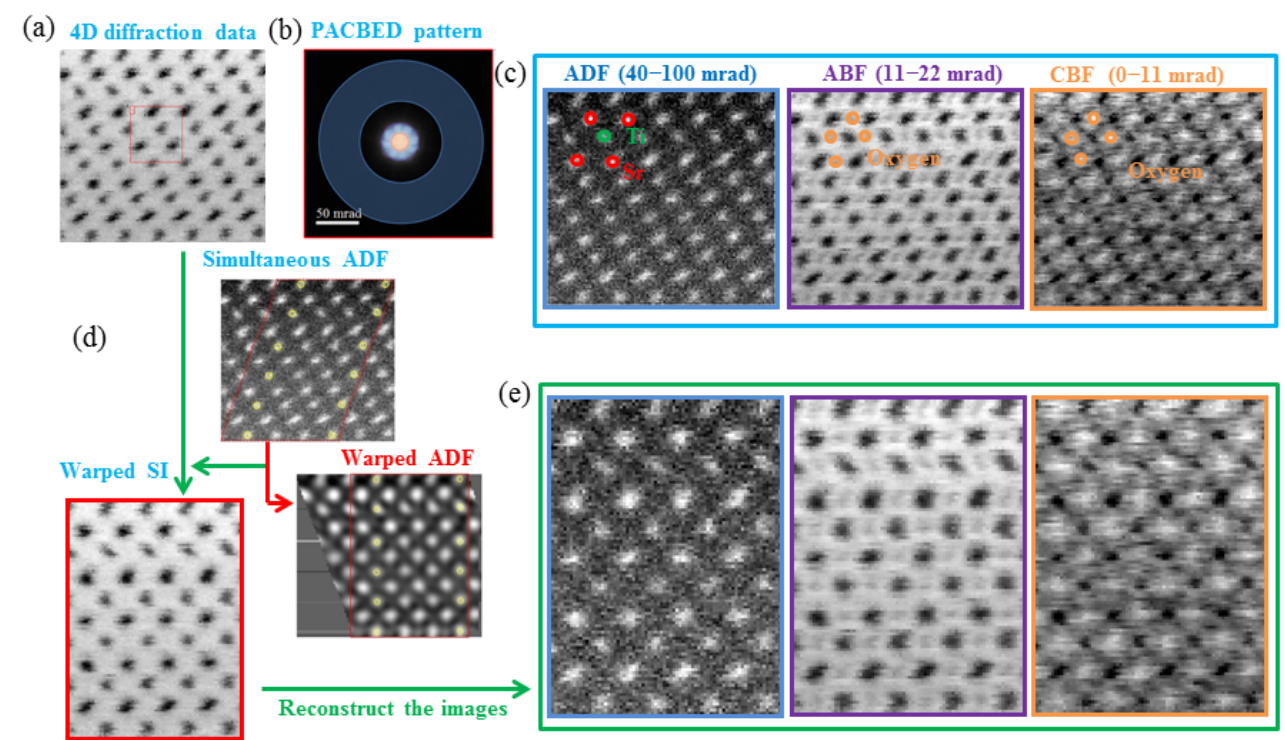

Figure 2. Correcting the distortion for 4D STEM diffraction imaging data. (a) The original DI data, (b) position-averaged CBED (PACBED) pattern overlaid by masks indicating radial integration ranges for later STEM image reconstruction, (c) $\mathrm{ADF}, \mathrm{ABF}$, and $\mathrm{CBF}$ images reconstructed from the data in (a). (d) Sketch of distortion correction for 4D diffraction imaging, distortion-corrected ADF image. (e) ADF, $\mathrm{ABF}$, and $\mathrm{CBF}$ images reconstructed from distortion-corrected 4D DI data. 